\title{
Investigation on the Mass Burning Rate of Biodiesel Blended with Ethanol Subjected to Cross Airflow
}

\author{
Mohd Rosdzimin Abdul Rahman ${ }^{*}$, Siti Noor Aliah Baharom², Hasan Mohd Faizal ${ }^{3}$ \\ ${ }^{1}$ Department of Mechanical Engineering, Faculty of Engineering, Universiti Pertahanan Nasional Malaysia, Kem Sg. Besi, \\ 57000, Kuala Lumpur, Malaysia \\ ${ }^{2}$ Engineering Research Centre, MARDI Headquaters, Serdang, Selangor, Malaysia \\ ${ }^{3}$ School of Mechanical Engineering, Faculty of Engineering, Universiti Teknologi Malaysia, Johor Baharu 81310, Johor, \\ Malaysia
}

Corresponding Author Email: rosdzimin@gmail.com

https://doi.org/10.18280/ijht.370208

Received: 4 August 2017

Accepted: 18 April 2018

\section{Keywords:}

biodiesel, burning rate, cross airflow, pool fire

\begin{abstract}
Burning rates of the biodiesel B7 and blended with $10 \%$ vol. and $15 \%$ vol ethanol in $50 \mathrm{~mm}$ diameter of pan were investigated. Angle of the pan wall was set to 0 deg. and $60 \mathrm{deg}$. and 1 $\mathrm{m} / \mathrm{s}$ to $3.5 \mathrm{~m} / \mathrm{s}$ of longitudinal air, flows across the pan. Results show that, as increase in the longitudinal airflow speed, the burning rates of biodiesel B $7+15 \%$ vol ethanol increased, but it decreased for the biodiesel B7 and biodiesel B7+10\%vol. ethanol. Moreover, burning rate of $0 \mathrm{deg}$. is higher than that $60 \mathrm{deg}$. for all cases. It is concluded that for the case of biodiesel $\mathrm{B} 7+15 \%$ vol ethanol, incoming air helps in increasing the burning rate. But for biodiesel B7 and biodiesel B7+10\%vol. ethanol, the incoming air reduces the pan temperature and leads to low burning rate. Furthermore, 0 deg. case gives a higher burning rate for all fuels because flame tilt significantly and increased the rate of heat transfer by conduction and radiation from the flame to the fuel. The $60 \mathrm{deg}$. case showed that flame is less tilted thus leads to less burning rate.
\end{abstract}

\section{INTRODUCTION}

Biodiesel is an alternative fuel to replace fossil fuel. In Malaysia, palm oil methyl ester is used as a biodiesel and currently, the biodiesel B7 (7 \% palm oil methyl ester and $93 \%$ diesel fuel) is used widely in Malaysia since 2014. Biodiesel was used in internal combustion engine and it performance and low emissions is proven $[1,2]$. Biodiesel is not only used alone, but it is also blended with ethanol. This type of fuel has been used in compression ignition engine without any changes and can minimize the used of diesel fuel by approximately 25-30 \% [3]. Yilmaz [4] studys the biodiesel + ethanol + diesel and biodiesel + methanol + diesel and found that the performance and emission characteristic are comparable to the diesel. In overall the $\mathrm{CO}$ and $\mathrm{HC}$ emission increased as increases in alcohol concentration and NO was decreased. Oliveira et al. [5] study the performance of biodiesel and ethanol in the lower specific fuel consumption and found that in cylinder peak pressure and heat release rate, brake specific fuel consumption increased with the use of ethanol. Moreover, $\mathrm{CO}_{2}, \mathrm{CO}$ and THC decreased. Performance of the biodiesel in the internal combustion engine is proven. Since the biodiesel is widely used in vehicle, safety factor regarding the fire accident at the storage need to be investigated. Fire accident due to liquid hydrocarbon in a process industry is the big issue of safety concerns. Basically, a fire in process industry is treated as a pool fire. The fire occurs in an enclosed or an open area.

The pool fire in an enclosed area is considered as a fire in a quiescent atmosphere while in an open area, the effect of cross airflow on fire cannot be neglected. Research work on pool fire in a quiescent atmosphere was done which targeted on the burning rate, the flame height and pulsation, the thermal radiation, the total kinetic energy, and the burnt gas emission [6-8]. Moreover, research work on pool fire that affected by cross airflow has been done [9-17]. Gasoline pool fire under cross airflow was studied [10] and found that the mass burning rate of gasoline at certain cross air velocity decreased with pool size and vice versa with quiescent air condition. Moreover, the increment in burning rate is influenced by the tilted fire. The ethanol pool fire under cross airflow was studied by Tao et al. [13] and observed that the burning rate increases as increased in airflow velocity. They also found that burning rate is sensitive to the airflow impingement angle. Jiang and $\mathrm{Lu} \mathrm{[16]} \mathrm{studied} \mathrm{the} \mathrm{burning}$ rate and flame tilted characteristics of acetone pool fire under cross airflow. They have seen that burning rate of acetone increased linearly with airflow velocity from $0-0.5 \mathrm{~m} / \mathrm{s}$ and started to decrease until airflow velocity of $1.5 \mathrm{~m} / \mathrm{s}$. At $1.5-2.5$ $\mathrm{m} / \mathrm{s}$, the mass burning rate increased gradually. $\mathrm{Hu}$ et al. [9] studied the burning rate of square and rectangular pool using gasoline and methanol under cross airflow. Burning rates of methanol and gasoline give a different response. The burning rate of methanol was decreased whilst for the case of $5 \mathrm{~cm}$ square pool the burning rate increased. Moreover, the burning rate of small pool increased significantly compared to larger pool.

Jiang and $\mathrm{Lu}$ [12] and Jiang and Lu [16] studied mass loss rates of circular pool using aviation fuel subjected to cross airflow. They found that the mass loss rates of the aviation pool fire varied nonlinearly with the cross airflow velocity. This behaviour is controlled by three mechanisms namely; 
thermal radiation control stage, radiation-convection control stage and convection control stage. Hu et al. [15] studied the heat feedback in medium pool fire with cross airflow and observed that heat transfer by conduction increased as increase in airflow velocity and prominent for small pool size compared to large pool size. Type of fuel is significantly influenced by heat feedback; heat conduction in heptane is more prominent compare to ethanol. Moreover, they found that heat transfer by radiation was nearly negligible at higher velocity of cross airflow. Tao et al. [17] studied the flame length of alcohol and n-heptane pool fire under cross airflow and found that the flame length increased with airflow velocity. The flame length influenced heat release rate directly and significantly influenced the combustion efficiency. Hu et al. [11] studied on the radiation feedback from heptane pool fire under cross airflow to the fuel surface and found that flame radiation feedback for cross airflow case was different from the quiescent condition case. This difference is because of the flame deflection. The flame deflection effect should be considered in scaling the burning behaviour of pool fire in cross airflows.

It is seen that there is no previous study on pool fire that used a biodiesel as a fuel. It is therefore important to study the pool fire biodiesel since it has been used widely. Thus, the objective of this work is to investigate the behaviour of biodiesel POME B7 and blended with ethanol subjected to cross airflow at different pan wall angles.

\section{EXPERIMENTAL WORKS}

Figure 1 shows the schematic diagram of the experimental setup. The aluminium circular pan with diameter of $50 \mathrm{~mm}$ was used. A $60^{\circ}$ chamfer was created at the top of pan wall (Figure 2). The horizontal cross air flow was generated by a blower with a distance of $L=45 \mathrm{~cm}$ from the pan. The cross airflow velocity considered in the experiments was $1.2 \mathrm{~m} / \mathrm{s}$ to $3.5 \mathrm{~m} / \mathrm{s}$. Kanomax 6006 hot wire annemometer with resolution $0.01 \mathrm{~m} / \mathrm{s}$ was used to measure the airflow velocity at the pan position. The pan was positioned horizontally on the wood to reduce heat loss from pan bottom. Images of the flame were taken using Casio Exilim EX-ZR200 High Speed digital camera and was located orthogonal to the direction of cross airflow. The pans were filled with the fuel and leaving a small gap between the fuel surface and the lip of the pan. This allowed for the volumetric expansion of liquid upon heating and prevented the spilling of the liquid from the pan. Fuels used in this study were biodiesel B7 and ethanol (Figure 3). The biodiesel and ethanol were mixed using magnetic strirer for 6 hours. The viscosity of the fuel was measure using Brookfield Rheometer and the viscosity is shown in Table 1. Initially, the biodiesel B7 and biodiesel B7+10 \%vol. ethanol was ignited by supplying an external heat source to the pan as to help in increasing the fuel temperature to the boiling point. After flame exists, the external heat was removed. Once the flame was stable, cross airflow was introduced. While the biodiesel B7+15\%vol. ethanol was ignited without the external heat source.



Figure 1. Schematic of the experimental setup



(a)

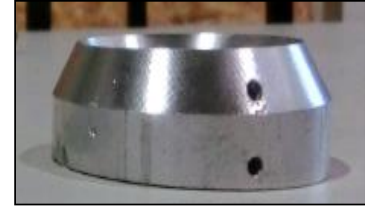

(b)
Figure 2. Fuel pan (a) $0^{\circ}$ and (b) $60^{\circ}$ pan wall angle



Figure 3. Picture of fuel (a) biodiesel B $7+15 \%$ vol. ethanol, (b) biodiesel B7+10\%vol. ethanol and (c) biodiesel B7

Table 1. The viscosity of the fuel

\begin{tabular}{cccc}
\hline \multirow{2}{*}{ Fuel } & \multicolumn{3}{c}{ Biodiesel B7 } \\
\cline { 2 - 4 } & $\begin{array}{c}\text { 0\%vol. } \\
\text { Ethanol }\end{array}$ & $\begin{array}{c}10 \% \text { vol. } \\
\text { Ethanol }\end{array}$ & $\begin{array}{c}15 \% \text { vol. } \\
\text { Ethanol }\end{array}$ \\
\hline $\begin{array}{c}\text { Viscosity } \\
\text { (Pa.s) }\end{array}$ & 0.0049 & 0.0024 & 0.0021 \\
\hline
\end{tabular}

\section{RESULTS AND DISCUSSION}

A deflection angle of the flame is an angle measured from the vertical line at the centre of the pan to the tip of the flame (Figure 4). 


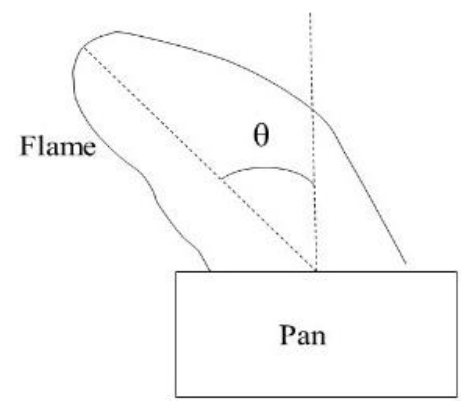

Figure 4. Schematic diagram of the flame deflection angle, $\theta$

Figure 5 shows the flame deflection angle for difference pan wall angle and cross airflow velocity.



(a)

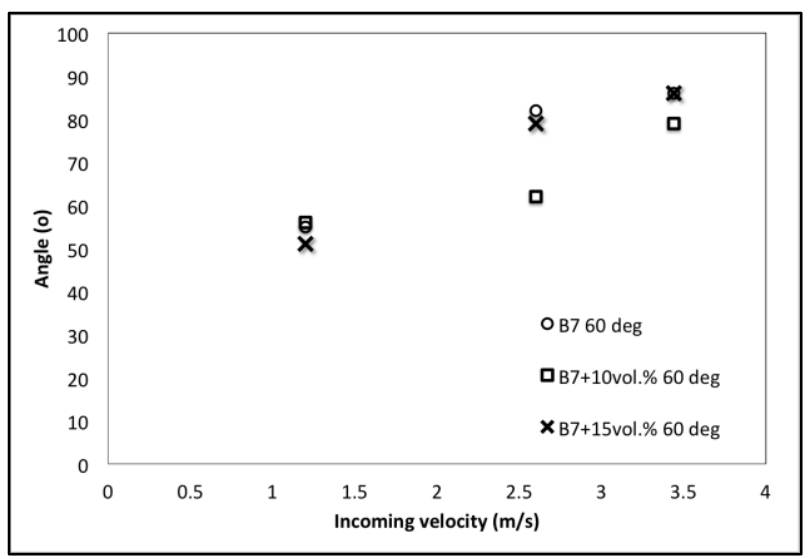

(b)

Figure 5. Flame deflection angle at various incoming cross airflow velocity for (a) $0^{\circ}$ and (b) $60^{\circ}$ wall pan edge

It can be seen that $0^{\circ}$ angle has a larger flame deflection compares to $60^{\circ}$ angle. This is due to the deflection of incoming airflow by the pan's wall edge, which affected the total volume of airflow towards the flame. Moreover, as the velocity of cross airflow increases, the deflection angle of flame increased. This shows that the velocity of the cross airflow is directly proportional to the deflection of the flame. Furthermore, for both cases $0^{\circ}$ and $60^{\circ}$, the deflection of flame is higher at the higher cross airflow velocity. This observation shows that the pan's wall angle is less significant toward flame deflection at higher cross airflow velocity. Figure 6 shows the mass burn rate of biodiesel B7, biodiesel B7+10 \%vol. ethanol and biodiesel B7+15\%vol. ethanol inside the pan with wall angle of $0^{\circ}$ and $60^{\circ}$.



(a)



(b)

Figure 6. Mass burning rate at various incoming cross airflow velocity for (a) $0^{\circ}$ and (b) $60^{\circ}$ wall pan edge

It is seen in all cases, biodiesel B7+10 \%vol. ethanol exhibited the highest mass burning rate followed by biodiesel B7 and biodiesel B7+15\%vol. ethanol. Higher mass burning rate of biodiesel $\mathrm{B} 7+10 \% \mathrm{vol}$. ethanol was because of the additional of an ethanol. By adding the ethanol into the biodiesel B7, the viscosity of fuel decreased (Table 1) which leads to decrease in boiling point and helped the fuel to ignite without additional heat source as compared to the biodiesel B7 (without an additional of ethanol). The biodiesel B7+15\%vol. ethanol gave the lowest mass burning rate because the ignition was started without heat addition to the pan. Thus, it gave lower mass burning rate. On the other hand, ignition of the biodiesel B7 and biodiesel B7+10 \%vol. ethanol was started by adding a heat to the pan. The heat was added to the pan as to increase the fuel temperature to the boiling temperature. The mass burning rate of $0^{\circ}$ angle (Figure 6(a)) for all fuels was higher than $60^{\circ}$ angle (Figure 6(b)). The effect of the pan wall angle toward mass burning rate can be explained based on the flame deflection angle as shown in Figures 5(a) and 5(b). Figures 5(a) and 5(b) show the flame deflection at various incoming cross airflow velocity for $0^{\circ}$ angle and $60^{\circ}$ angle. For all fuels, the flame deflection increases as the incoming cross airflow velocity increases. Meanwhile the flame deflection decrease as the pan wall increases. At $0^{\circ}$ angle, deflection of flame was larger and it correlated to the higher mass burning rate 
depicted in Figures 6(a) and 6(b). On the other hand, the lower mass burning rate was correspond to low angle of the flame deflection at $60^{\circ}$ angle. Thus, the more flame deflected toward the pan, the higher the heat transfer rate.

A relation between the flame deflection and the mass burning rate is connected with a heat transfer mechanism. Heat transfer mechanism from the flame can be in radiation and conduction. If the flame far from the pan, the heat is transfer by radiation only and if the flame close to the pan the heat is transferred by radiation and conduction. Combination of heat transfer mechanism by radiation and conduction gives a larger heat transfer rate to the pan. Larger heat transfer rate to the pan increases the fuel temperature and leads to higher mass burning rate. This observation is corresponded to the expression by Hottel [18] on the rate of heat feedback as,

$$
\dot{m}^{\prime \prime}=\frac{4 k\left(T_{F}-T_{B}\right)}{D}+h\left(T_{F}-T_{B}\right)+\sigma F\left(T_{F}^{4}-T_{B}^{4}\right)\left(1-e^{\kappa \beta D}\right)
$$

Equation (1) indicated that the mass burning rate is determined by the heat feedback to the pan. The larger heat feedback the greater mass burning rate.

Moreover, it is interesting to observe that the mass burn rate of biodiesel B7 and biodiesel B7+10 \%vol. ethanol decreased as increases in cross airflow velocity for all pan wall angle cases. While, the mass burning rate of biodiesel B7+15\%vol. ethanol increases as the cross airflow velocity increases for all pan wall angle. Increases in mass burning rate of biodiesel $\mathrm{B} 7+15 \%$ vol. ethanol when the cross airflow velocity increased was predicted. It is because more air was introduced to the fire zone and mixed with the fuel. This observation is agreed with $\mathrm{Hu}$ et al. [10].

Later, an interesting behavior of the mass burning rate of the biodiesel B7 and biodiesel B7+10 \%vol. ethanol is supposed due to cooling effect towards the pan by the incoming cross airflow. The biodiesel B7 and biodiesel B7+10\%vol. ethanol is sensitive to the heat because of their higher boiling temperature. This factor is strengthened by observing the Figures 5(a), 5(b), 6(a) and 6(b). These figures show that flame is less deflected at $60^{\circ}$ angle as compared to $0^{\circ}$ angle. Moreover, at higher incoming cross airflow velocity the mass burning rate decreases and very significant for $60^{\circ}$ angle case. While, less deflected flame transferred less heat to the pan and higher incoming cross airflow velocity bring the cooling effect to the pan and leads to decreased in the mass burning rate.

\section{CONCLUSIONS}

The objectives of this study were achieved. In this study, the conclusions are;

- Pan wall angle has an effect on flame deflection and the mass burn rate.

- At small pan wall angle, the deflection of flame is large compared to bigger angle due to the deflection airflow towards the flame.

- Biodiesel B7+10 \%vol. ethanol gives the highest mass burning rate followed by biodiesel B7 and biodiesel B7+15\%vol. ethanol. This is because of the addition of an ethanol to the biodiesel reduces the viscosity and lead to low boiling temperature.

- Biodiesel B7+15\%vol. ethanol shows a predicted mass burning rate behavior as increases in incoming cross airflow velocity.

- Biodiesel B7 and biodiesel B7+10 \%vol. ethanol flame shows an interesting behavior. Decrease in mass burning rate as increased in incoming cross airflow velocity and pan wall angle is supposed due to the cooling effect towards the fuel pan.

\section{REFERENCES}

[1] Xue, J., Grift, T.E., Hansen, A.C. (2011). Effect of biodiesel on engine performances and emissions. Renewable and Sustainable Energy Reviews, 15(2): 1098-1116. https://doi.org/10.1016/j.rser.2010.11.016

[2] Qadri, U., Marouf Wani, M. (2019). Experimental investigation on multi-cylinder SI engine fueled conventional gasoline, ethanol blends, and microemulsion as an alternative fuel. Mathematical Modelling of Engineering Problems, 6(1): 69-76. https://doi.org/10.18280/mmep.060109

[3] Shahir, S.A., Masjuki, H.H., Kalam, M.A., Imran, A., Fattah, I.M.R., Sanjid, A. (2014). Feasibility of dieselbiodiesel-ethanol/bioethanol blend as existing CI engine fuel: An assessment of properties, material compatibility, safety and combustion. Renewable and Sustainable Energy Reviews, 32: 379-395. https://doi.org/10.1016/j.rser.2014.01.029

[4] Yilmaz, N. (2012). Comparative analysis of biodieselethanol-diesel and biodiesel-methanol-diesel blends in a diesel engine. Energy, 40(1): 210-213. https://doi.org/10.1016/j.energy.2012.01.079

[5] de Oliveira, A., Valente, O., Sodre, J. (2016). Performance of a diesel engine operating with blends of diesel, biodiesel and ethanol in the lower specific fuel consumption range. SAE Technical Paper Series, 201636-0160. https://doi.org/10.4271/2016-36-0160

[6] Kamikawa, D., Weng, W.G., Kagiya, K., Fukuda, Y., Mase, R., Hasemi, Y. (2005). Experimental study of merged flames from multifire sources in propane and wood crib burners. Combustion and Flame, 142: 17-23. https://doi.org/10.1016/j.combustflame.2005.02.004

[7] Steinhaus, T., Welch, S., Carvel, R.O., Torero, J.L. (2007). Large-scale pool fires. Thermal Science, 11(2): 101-118. https://doi.org/10.2298/TSCI0702101S

[8] Vali, A., Nobes, D.S., Kostiuk, L.W. (2013). Effects of altering the liquid phase boundary conditions of methanol pool fires. Experimental Thermal and Fluid Science, 44: 786-791. https://doi.org/10.1016/j.expthermflusci.2012.09.023

[9] Hu, L.H., Liu, S., Peng, W., Huo, R. (2009). Experimental study on burning rates of square/rectangular gasoline and methanol pool fires under longitudinal airflow in a wind tunnel. Journal of Hazardous Materials, 169(1-3): 972-979. https://doi.org/10.1016/j.jhazmat.2009.04.050

[10] Hu, L.H., Liu, S.A., Xu, Y., Li, D. (2011). A wind tunnel experimental study on burning rate enhancement behavior of gasoline pool fires by cross airflow. Combustion and Flame, 158(3): 586-591. https://doi.org/10.1016/j.combustflame.2010.10.013

[11] Hu, L.H., Liu, S., Wu, L. (2013). Flame radiation feedback to fuel surface in medium ethanol and heptane pool fires with cross air flow. Combustion and Flame, 160(2):

295-306. 
https://doi.org/10.1016/j.combustflame.2012.10.016

[12] Jiang, P., Lu, S. (2013). Effects of cross air flow on mass loss rates of circular aviation fuel pool fires in large open space. Procedia Engineering, 62: 309-316. https://doi.org/10.1016/j.proeng.2013.08.069

[13] Tao, C., He, Y., Li, Y., Wang, X. (2013). Effects of oblique air flow on burning rates of square ethanol pool fires. Journal of Hazardous Materials, 260: 552-562. https://doi.org/10.1016/j.jhazmat.2013.06.015

[14] Tang, F., Li, L.J., Zhu, K.J., Qiu, Z.W., Tao, C.F. (2015). Experimental study and global correlation on burning rates and flame tilt characteristics of acetone pool fires under cross airflow. International Journal of Heat and Mass Transfer, 87: 369-375. https://doi.org/10.1016/j.ijheatmasstransfer.2015.04.019

[15] Hu, L., Hu, J., Liu, S., Tang, W., Zhang, X. (2015) Evolution of heat feedback in medium pool fires with cross air flow and scaling of mass burning flux by a stagnant layer theory solution. Proceeding of the Combustion Institute, 35: 2511-2518. https://doi.org/10.1016/j.proci.2014.06.074

[16] Jiang, P., Lu, S. (2016). Pool fire mass burning rate and flame tilt angle under crosswind in open space. Procedia Engineering, 135:

261-274. https://doi.org/10.1016/j.proeng.2016.01.122

[17] Tao, C., Wang, X., Ma, P. (2017). An experimental investigation of flame length of alcohol and N-heptane pool fires under air cross condition. Experimental Heat Transfer, $30(6)$ :

489-499. https://doi.org/10.1080/08916152.2017.1305468

[18] Blinov, V.I., Khudiakov, G.N., Hottel, H.C. (1959). Certain laws governing diffusive burning of liquids. Fire Research Abstracts and Reviews, 1: 41-44.

\section{NOMENCLATURE}

$D \quad$ diameter of the fuel pan, $[\mathrm{mm}]$

$F \quad$ view factor

$H \quad$ height of the fuel pan, [mm]

$h \quad$ heat transfer coefficient, $\left[\mathrm{W} / \mathrm{m}^{2} \mathrm{~K}\right]$

$k$ thermal conductivity, [W/mK]

$L \quad$ distance from ducting exit to fuel pan, [m]

$\dot{m}^{\prime \prime} \quad$ mass burning rate

$T$ temperature $[\mathrm{K}]$

\section{Greek}

$\alpha \quad$ fuel pan wall angle, [deg]

$\theta \quad$ flame deflection angle, $[\mathrm{deg}]$

$\kappa \beta \quad$ extinction ceofficient

\section{Subscript}

$B \quad$ pan 